\title{
Bi-metal coated aperture SNOM probes
}

\author{
Tomasz J. Antosiewicz ${ }^{a, b, \dagger}$, Piotr Wróbel, ${ }^{c, \ddagger}$ and Tomasz Szoplik ${ }^{c}$ \\ ${ }^{a}$ Interdisciplinary Centre for Mathematical and Computational Modelling, University of \\ Warsaw, Pawinskiego 5A, 02-106 Warsaw, Poland; \\ ${ }^{b}$ Departament of Applied Physics, Chalmers University of Technology, SE-412 96 Göteborg, \\ Sweden; \\ ${ }^{c}$ Faculty of Physics, University of Warsaw, Pasteura 7, 02-093 Warszawa, Poland
}

\begin{abstract}
Aperture probes of scanning near-field optical microscopes (SNOM) offer resolution which is limited by a sum of the aperture diameter at the tip of a tapered waveguide probe and twice the skin depth in metal used for coating. An increase of resolution requires a decrease of the aperture diameter. However, due to low energy throughput of such probes aperture diameters usually are larger than $50 \mathrm{~nm}$. A groove structure at fiber core-metal coating interface for photon-to-plasmon conversion enhances the energy throughput 5-fold for $\mathrm{Al}$ coated probes and 30-fold for Au coated probes due to lower losses in the metal. However, gold coated probes have lower resolution, first due to light coupling from the core to plasmons at the outside of the metal coating, and second due to the skin depth being larger than for Al. Here we report on the impact of a metal bilayer of constant thickness for coating aperture SNOM probes. The purpose of the bilayer of two metals of which the outer one is aluminum and the inner is a noble metal is to assure low losses, hence larger transmission. Using body-of-revolution finite-difference time-domain simulations we analyze properties of probes without corrugations to measure the impact of using a metal bilayer and choose an optimum bi-metal configuration. Additionally we investigate how this type of metalization works in the case of grooved probes.
\end{abstract}

Keywords: Scanning near-field optical microscopy, SNOM, FDTD, surface plasmon, nanoaperture

\section{INTRODUCTION}

Optical device minutarization requires measurement and fabrication tools for production, inspection and characterization at the nanoscale. Among these tools are nano-photonic structures for guiding and trapping of light, plasmonic sensors for bio- and chemical sensing, field enhancing structures for nanolithography and others. Device inspection requires nanometer size resolution which can be achieved with atomic force and scanning tunneling microscopy in vacuum, but not so under normal conditions with visible light for which these structures are designed. At visible frequencies e.g. confocal and scanning probe microscopy are capable of analyzing samples at resolution better than a plasmon wavelength. Of particular interest is the scanning optical near-field microscope $(\mathrm{SNOM})^{1}$ with aperture probes, as it may be used for measurements of electric and/or magnetic ${ }^{2,3}$ field components in $e . g$. photonic crystal cavities, ${ }^{4,5}$ optical imaging of biological samples, ${ }^{6}$ direct measurements of surface plasmon-polaritons (SPPs), ${ }^{7}$ nano-scale Raman and IR spectroscopy for chemical analysis, ${ }^{8}$ optical trapping ${ }^{9-11}$ and others.

Aperture probes have a tapered dielectric core and are covered by a thin metal layer save for its tip, thus forming an aperture. The reason for metalization is light confinement, as otherwise the electromagnetic field would leak out of the dielectric core. In a metal-clad cylindrical dielectric waveguide light may propagte only if the radius is large enough and as soon as it falls below a certain value - the cut-off diameter - the imaginary part of the effective refractive index grows to large values and the fields experience an exponential decrease untill they reach the aperture. However, by the time they reach the end of the probe the fields fall to values on the order of $10^{-5}$ and typically smaller than $10^{-7}$. Of course, the smaller the aperture is (thus better resolution), the weaker is the emission.

\footnotetext{
${ }^{\dagger}$ tantos@igf.fuw.edu.pl, tomasza@chalmers.se, ${ }^{\ddagger}$ piotr.wrobel@igf.fuw.edu.pl
} 

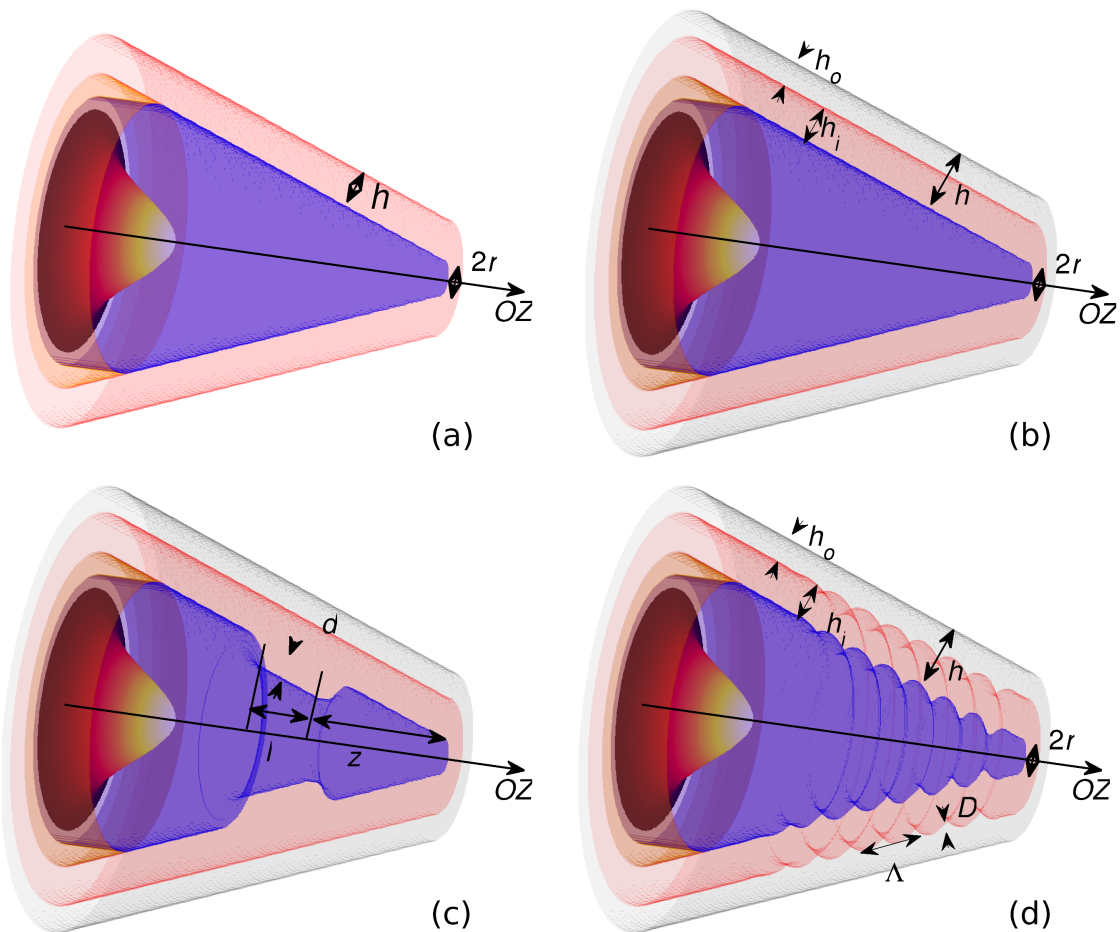

Figure 1. Models of aperture probes for SNOM: (a) commercially available aluminum coated with aperture radius $r$, coating thickness $h$; (b) bi-metal coated probe with inner $h_{i}$ and outer $h_{o}$ thick layers; (c) bimetal coated probe with one groove of dimension $l+2 d$ by $d$ located at position $z$ from the aperture; (d) bi-metal coated probe with sinusoidal grating of period $\Lambda$ and modulation $D$ similar to ones fabricated using a modified Turner method.

Resolution $\Delta r$ offered by SNOM is determined by two factors, namely the radius of the aperture $r$ - over which we have some degree of control - and the skin depth $a$ defined as a distance over which the electric field decreases by $e$ in metal and is the metal's inherent property. Thus, $\Delta r=2 r+2 a$. According to measurements by Wang et al. ${ }^{12}$ of silver films the skin depth is almost constant in the NIR and the long wavelength part of the visible spectrum and is slightly larger than $10 \mathrm{~nm}$. Aluminum, due to higher losses and a larger negative real part of permittivity, has a smaller skin depth than Ag and thus is the metal of choice of SNOM probe coating. As the skin depth depends on the material paremeters of the coating layer the way of improving SNOM resolution leads via an aperture decrease. However, high resolution SNOM probes with apertures smaller than $50 \mathrm{~nm}$ are characterized by very low energy throughput, what requires an enhancement in energy throughput before increasing resolution. Recent examples of such pursuits include e.g. work by Imura et al. who report anomalous light transmission when a gold disk is placed in front of subwavelength apertures. ${ }^{13}$ Previously developed ways of achieving this condition include modification of the taper angle, ${ }^{14}$ nanopatterning the aperture area into bow-ties ${ }^{15}$ and I-shapes ${ }^{16}$ or structuring the core-coating interface to facilitate plasmon excitation ${ }^{17-20}$ using noble metals as coating.

The use of noble metals for lower losses in corrugated plasmonic SNOM probes ${ }^{20}$ allows for large transmission enhancement reaching 5 and 30 times that of classical probes for Al and Au coatings, respectively. A downside to using coating metals like silver or gold is that due to a larger skin depth the resolution decreases a little compared to Al-coating or, in the worst case, light tunneling through the coating may be large enough to outweigh te signal emitted by the aperture. In the latter situation the resolution decreases to the diameter of the outer metal-air interface. Thus, corrugated plasmonic SNOM probes have a trade-off between transmission enhancement and resolution decrease due to a larger skin depth.

In this technical report we consider the impact of a metal bilayer of constant thickness for coating aperture SNOM probes. The bilayer consists of two metals of which the outer one is aluminum and the inner is a 
noble metal for assuring low losses, hence larger transmission. Using body-of-revolution finite-difference timedomain simulations (BOR FDTD) we analyze transmission spectra of probes without corrugations to measure the impact of using a metal bilayer. Next, we investigate how this type of metalization works in the case of grooved probes.

\section{APERTURE SCANNING NEAR-FIELD OPTICAL MICROSCOPE PROBES}

An unmodified SNOM probe is modeled as a dielectric cone with a half-angle $\alpha=20^{\circ}$ with a varying aperture diameter $2 r$. In simulations we neglect the dielectric cladding an focus on photon-plasmon coupling in the tapered single mode optical fiber of $3 \mu \mathrm{m}$ core diameter. The core is considered to be dispersionless with permittivity $\epsilon=2.1$ and is coated with a single metal layer $h \mathrm{~nm}$ thick. Such a basic structure is shown in Fig. 1a. The first modification we introduce is a change of the sinlge layer into two of thicknesses $h_{i}$ and $h_{o}$, inner and outer respectively, keeping the total thickness constant $\left(h=h_{i}+h_{o}\right)$. The outer metal is always aluminum and the inner layer may be silver, gold or copper and its thickness is varied from zero to $h$. A probe with an intermediate values of $h_{i}$ and $h_{o}$ is shown in Fig. $1 \mathrm{~b}$.

The bi-metal coating is applied also to corrugated probes. In our work we consider two types of corrugated structures: with a single oval groove ${ }^{20}$ that may be milled in the core using focused ion beam or a set of quasi-sinusoidal grooves shown in Fig. 1c and d, respectively. The first type of structured probes is defined by three parameters: position $z$ relative to the aperture, length $l$ and depth $d$, with $l$ and $z$ measured along the axis of symmetry $O Z$. The sinusoidal grating of the second is determined by its period $\Lambda$ and modulation depth of $D=50 \mathrm{~nm}$.

\section{FINITE-DIFFERENCE TIME-DOMAIN SIMULATIONS}

We calculate the properties of bi-metal aperture SNOM probes without and with corrugations using the FDTD method, ${ }^{21}$ which allows for treatement of virtually any structure. However, this comes at the price of very large ccomputer resource requirements when simulating three-dimensional structures. There are many tricks that may be used to lessen needed memory space, for example unstructured grids, adaptive grids. Here, we take advantage of the fact that the probes have cylindrical symmetry, what enables us to use the Body of Revolution formalism to reduce the simulation two dimensions in the longitudinal and radial directions. We expand the electric $\vec{E}(r, \phi, z, t)$ and magnetic $\vec{H}(r, \phi, z, t)$ fields in the azimuthal direction $\phi$ in a Fourier series

$$
\begin{aligned}
& \vec{E}(r, \phi, z, t)=\sum_{m=0}^{\infty} \vec{E}_{c, m}(r, z, t) \cos m \phi+\sum_{m=0}^{\infty} \vec{E}_{s, m}(r, z, t) \sin m \phi, \\
& \vec{H}(r, \phi, z, t)=\sum_{m=0}^{\infty} \vec{H}_{c, m}(r, z, t) \cos m \phi+\sum_{m=0}^{\infty} \vec{H}_{s, m}(r, z, t) \sin m \phi,
\end{aligned}
$$

where $m$ is the azimuthal mode number. This analytical dependence on $m$ is incorporated into the FDTD code and to calculate the full properties one needs to calculate the interaction of subsequent decomposition elements with the probe. Fortunately, a Gaussian beam has only one nonzero element of the Fourier decomposition for $m=1$. We use an in-house code ${ }^{22}$ with Drude and Drude-Lorentz dispersion models and a convolution Perfectly Matched Layer ${ }^{21}$ to absorb scattered radiation. Use of the FDTD method allows for computation of the whole desired spectrum in one simulation as a result of using an ultrashort pulse as an excitation signal and utilization of the Fourier Transform (FT).

We use aluminum, copper, silver, and gold as coating materials. The Drude model is used for $\mathrm{Al}$ and $\mathrm{Ag}$, omitting the third term in Eq. 3, while the Drude-Lorentz model is employed for $\mathrm{Cu}$ and Au to describe their dispersion characteristics, especially increase of absorption due to interband transitions

$$
\epsilon(\omega)=\epsilon_{\infty}-\frac{\omega_{p}^{2}}{\omega(\omega+i \Gamma)}+\frac{\Delta \epsilon_{l} \omega_{l}^{2}}{\omega_{l}^{2}-2 i \omega \delta-\omega^{2}},
$$

with parameters listed in Table 1. The parameters are fitted to experimental data from Johnson and Christy ${ }^{23}$ for copper, silver, and gold and from Ordal et al. ${ }^{24}$ for aluminum for wavelengths $\lambda$ in the range $400-700 \mathrm{~nm}$. 


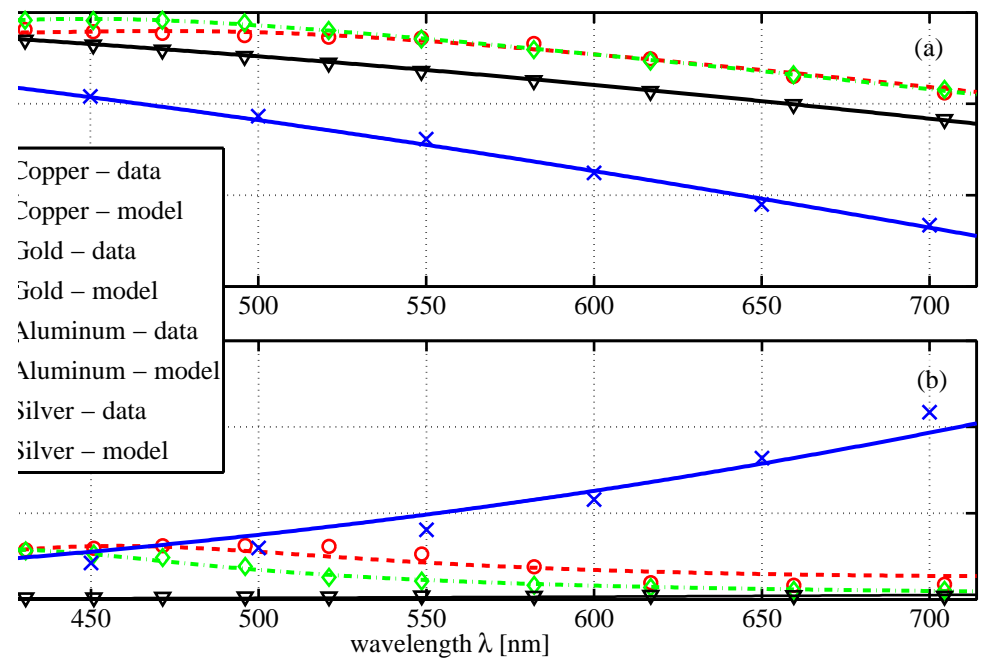

Figure 2. (a) Real $\Re(\epsilon)$ and (b) imaginary $\Im(\epsilon)$ parts of permittivity values for copper in red, gold in green, and aluminum in blue. Markers show experimental data from Johnson and Christy ${ }^{23}$ and Ordal et al., ${ }^{24}$ lines show fitted dispersion curves.

In Fig. 2 we present experimental data points taken from the two papers along with our calculated dispersion curves.

\section{PROPERTIES OF UNSTRUNCTURED BI-METAL COATED PROBES}

We analyse two parameters of SNOM probes: transmission efficiency and resolution calculated as the FWHM of the emitted radiation. Both parameters are measured $10 \mathrm{~nm}$ from the aperture where we record the temporal EM-field evolution and using the FT calculate radial profiles in the wavelength range of 400-700 $\mathrm{nm}$. The raw data is processed to show transmission enhancement of bi-metal coated probes over those coated only with aluminum. We define normalized transmission (a.k.a. transmission enhancement) as $\left.\mathcal{T}\right|_{x}$

$$
\left.\mathcal{T}\left(\lambda ; h_{i}\right)\right|_{x}=\frac{\left.T\left(\lambda ; h_{i}\right)\right|_{x}}{\left.T_{0}(\lambda)\right|_{\mathrm{Al}}}
$$

where $\left.T_{0}(\lambda)\right|_{\mathrm{Al}}$ is a reference transmission through a pure Al-coated probe, $\left.T\left(\lambda ; h_{i}\right)\right|_{x}$-transmission through a bi-metal probe with inner layer thickness $h_{i}$, and $x$ indicates the inner metal $\mathrm{Ag}, \mathrm{Cu}, \mathrm{Au}$. This procedure is carried out for a varying split of the total thickness $h$ into the inner $h_{i}$ and outer $h_{o}$ layers. Unless noted otherwise, the normalization is always carried out for the same aperture radius, i.e. $T$ and $T_{0}$ are calculated for probes with the same aperture size.

Table 1. Drude and Drude-Lorentz model parameters of metals used in simulations.

\begin{tabular}{ccccc}
\hline \multicolumn{4}{c}{ material } \\
\hline & aluminum & copper & gold & silver \\
\hline$\epsilon_{\infty}$ & 4.39 & 6.34 & 7.7 & 4.61 \\
$\omega_{p} / 2 \pi[\mathrm{THz}]$ & 3282.7 & 2195.4 & 2242.6 & 2260.6 \\
$\Gamma / 2 \pi[\mathrm{THz}]$ & 160.6 & 16.7 & 0.001 & 7.88 \\
$\Delta \epsilon_{l}$ & & 2.62 & 2.105 & \\
$\omega_{l} / 2 \pi[\mathrm{THz}]$ & & 674.34 & 720.02 & \\
$\delta / 2 \pi[\mathrm{THz}]$ & & 151.04 & 131.94 & \\
\hline
\end{tabular}



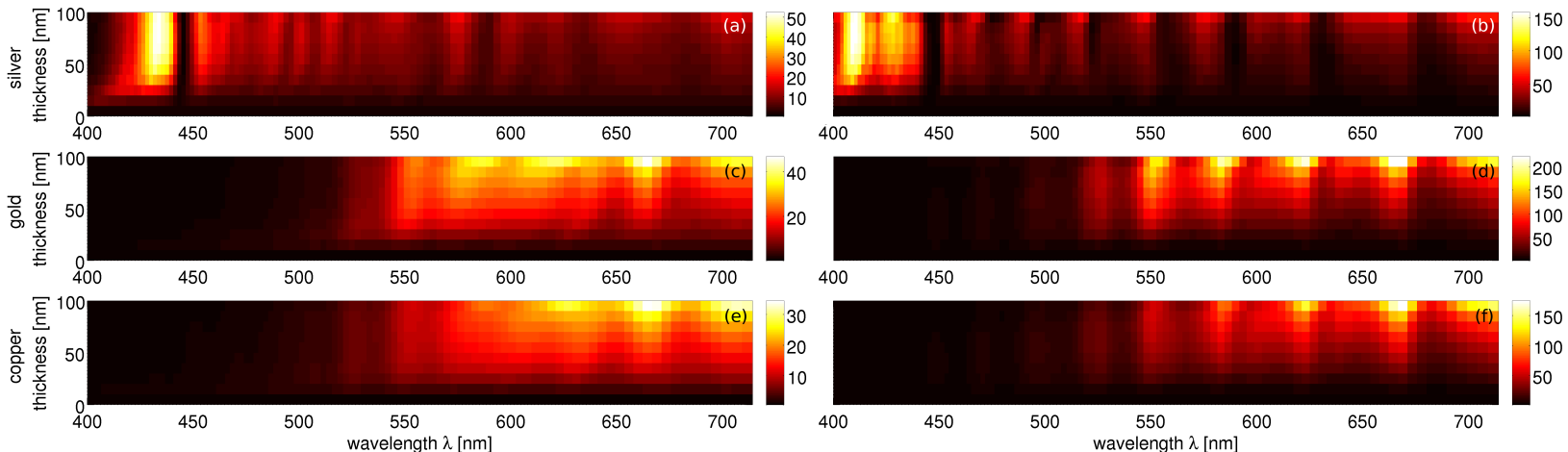

Figure 3. Transmission enhancement in bi-metal coated probes as function of inner metal type and thickness $h_{i} \in[0 ; 100] \mathrm{nm}$ for wavelengths $\lambda \in[400 ; 700] \mathrm{nm}$. Aperture radius is $25 \mathrm{~nm}$ in (a), (c), (e) and $15 \mathrm{~nm}$ in (b), (d), (f). The inner layer is silver for (a) and (b), gold for (c) and (d), and copper for (e) and (f).
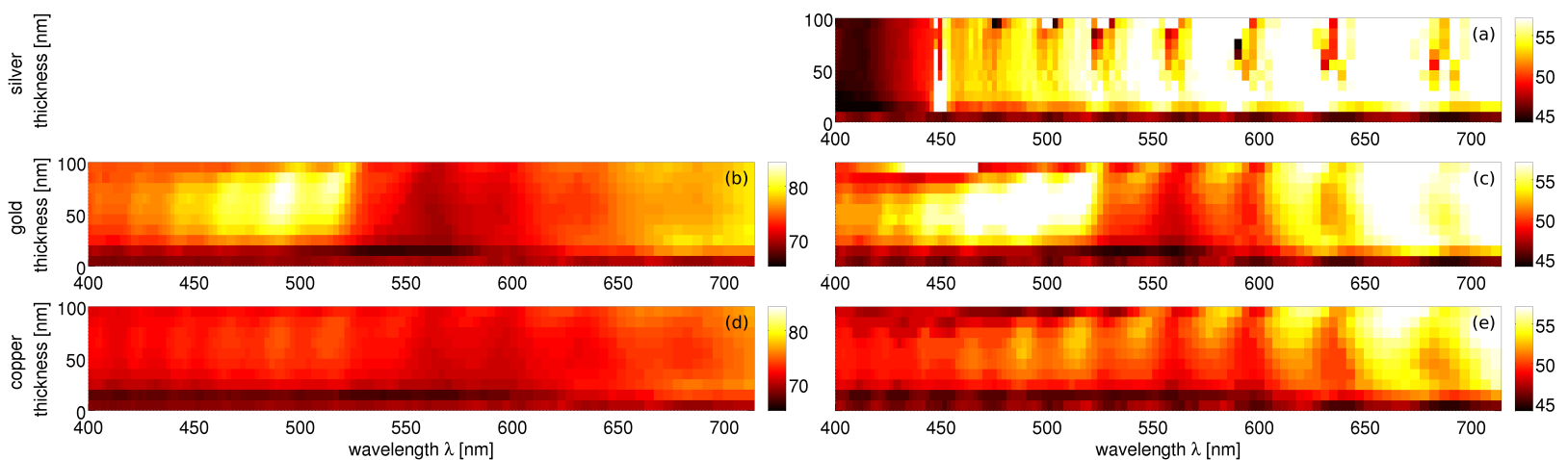

Figure 4. Resolution of bi-metal coated probes as function of inner metal type and thickness $h_{i} \in[0 ; 100] \mathrm{nm}$ for wavelengths $\lambda \in[400 ; 700] \mathrm{nm}$. Aperture radius is $25 \mathrm{~nm}$ in (b), (d) and $15 \mathrm{~nm}$ in (a), (c), (e). The inner layer is silver for (a), gold for (b) and (c), and copper for (d) and (e).

Figure 3 shows the transmission enhancement from using an increasing thickness of the inner nobel metal thickness for two aperture diameters. As expected, a metal with lower losses than aluminum increases the energy efficiency and this enhancement is larger for smaller apertures. We also note that a Fabry-Perot interference pattern of plasmon waves can be observed in the enhancement maps. This resonance is more pronounced for the smaller aperture and intensifies as the inner metal thickness increases. Also, subsequent maxima and minima are located at the same wavelengths for both radii. Plasmons launched on the metal surface surrounding the aperture are partially reflected back at the end of the coating and to a lesser extent at the metal interfaces reinforcing the fields in the aperture. This effect naturally depends on the total metal thickness which dictates the phase of the returning wave and interference with the field incident from the inside of the probe. For probes with an increasing noble metal content plasmons experience a decrease in loss and the interference becomes increasingly stronger. The interference is also visible in the resolution maps in Fig. 4. The decrease of the aperture diameter increases resolution by about $20 \mathrm{~nm}$ for a pure Al coated probe which has high losses (bottom line of all subplots for thickness $0 \mathrm{~nm}$ ) and offers negligible transmission for $r=15 \mathrm{~nm}$. Use of nobel metals increases transmission by up to 200 times, but at the same time decreases resolution. The best results are obtained for a copper inside layer which increases the spot size by only $5 \mathrm{~nm}$ compared to a pure aluminum probe over a wide wavelength range, while probes with silver and gold can only be used in narrower ranges. However, for probes tailor-made for selected wavelengths they offer a larger transmission enhancement than copper.

\section{PROPERTIES OF CORRUGATED BI-METAL COATED PROBES}

The analysis of corrugated bi-metal coated probes is carried out in a similiar way as for unstructured ones with one major difference. In this section we look only at probes with a decreased aperture radius $(r=15$ 

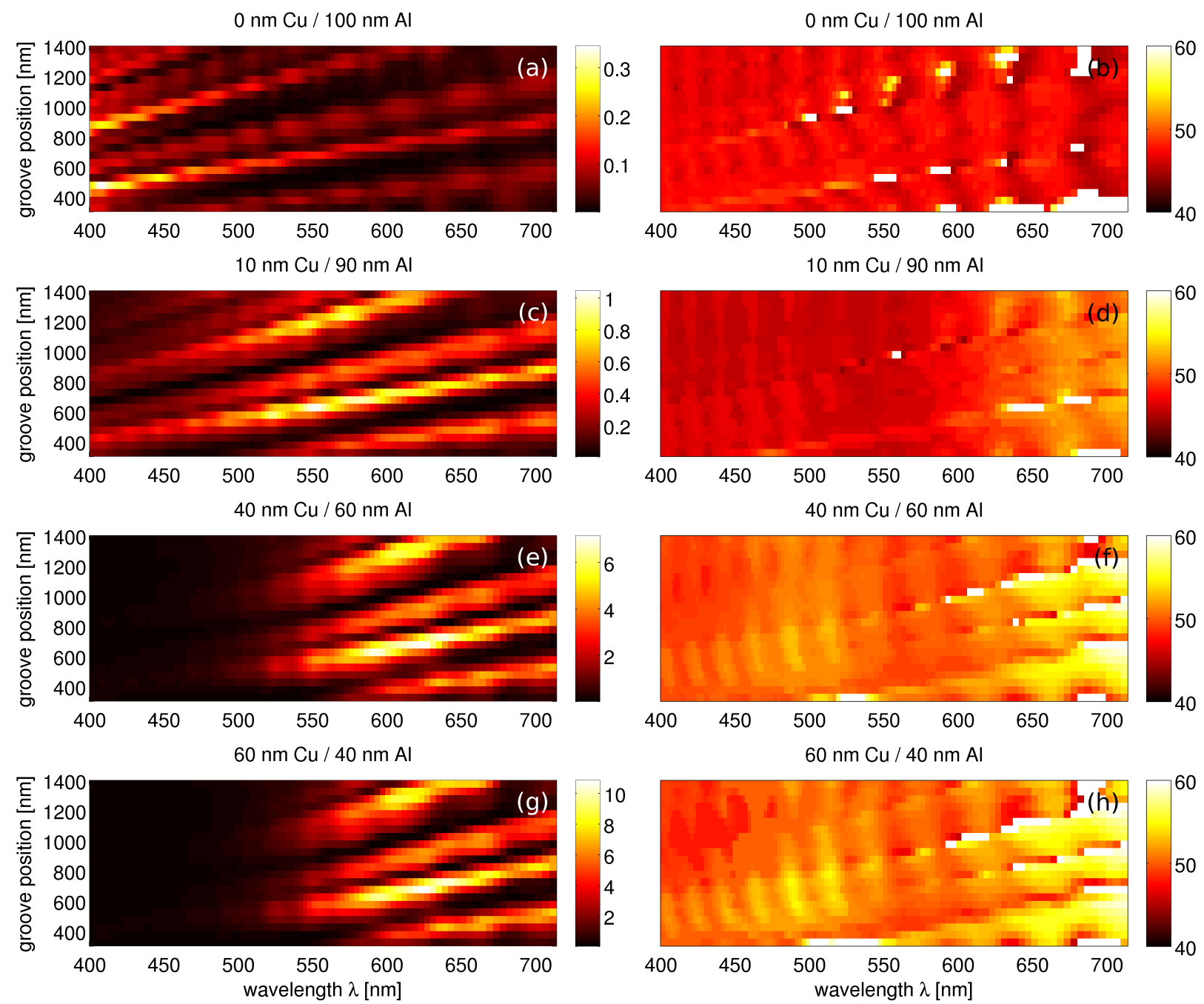

Figure 5. Transmission (a), (c), (e), (g) and resolution (b), (d), (f), (h) of Cu/Al coated probes $(r=15 \mathrm{~nm})$ with one groove as function of groove position $z$ for wavelengths $\lambda \in[400 ; 700] \mathrm{nm}$. Copper thickness $h_{i}$ is $0 \mathrm{~nm}$ in (a), (b), $10 \mathrm{~nm}$ in (c), (d), $40 \mathrm{~nm}$ in (e), (f), and $60 \mathrm{~nm}$ in (g), (h). Transmission is normalized to that of a pure $\mathrm{Al}$ coated unstructured $r=25 \mathrm{~nm}$ probe.

$\mathrm{nm}$ ), but normalize transmission to that of a pure $\mathrm{Al}$ coated $50 \mathrm{~nm}$ diameter probe. This is because we want to show that a probe with better resolution can have transmission comparable or even larger than that of a probe with worse resolution. We are interested in probes capable of working in a wide spectral range, so based on the results presented in the previous section we choose copper as the inner metal for further analysis. Also, we analyze a wide range of parameters and show results only for selected cases. To the main scanned parameter - the inner metal thickness - we add one additional for each of the two types of corrugated cores. In the case of probes with one groove we vary the position $z$ of the groove relative to the position of the aperture from 300 to $1400 \mathrm{~nm}$ with a step of $50 \mathrm{~nm}$ and for probes with a sinusoidal grating its lattice period $L$ from 700 to $1200 \mathrm{~nm}$ with a step of $50 \mathrm{~nm}$.

\subsection{Probes with one groove}

A full analysis of enhancement and resolution of single-grooved probes with a uniform metal coating was described by us elsewhere ${ }^{20}$ and will be very briefly recalled here. The groove scatteres light inside the probe into plasmons which propagate towards the aperture and this scattering is most effective when $z$ is matched 
to the cut-off, so optimum $z$ depends on the wavelength in terms of scattering efficiency. Depending on the groove-aperture separation $z$ a standing wave may form increasing or decreasing transmission. Also, $z$ determines cumulative losses of plasmons propagating to the aperture from the groove and the best position is determined by a trade-off between metal dispersion and propagation length.

In Fig. 5 we present transmission enhancement and resolution obtained from simulations for a copper inner metal layer for a probe with an aperture radius $r=15 \mathrm{~nm}$. In the first row the inner layer is zero i.e. the coating is made of pure aluminum. The enhancement trend is similar to that in our previous paper, ${ }^{20}$ although here the transmission values are lower due to normalization versus a $r=25 \mathrm{~nm}$ probe. As the copper layer increases, the transmission enhancement increases very quickly even for thin layers reaching 1 for $10 \mathrm{~nm}$ of $\mathrm{Cu}, 7$ for $40 \mathrm{~nm}$ of $\mathrm{Cu}$, and 11 for $60 \mathrm{~nm}$ of $\mathrm{Cu}$. Also, a redshift of maximum enhancement is observed which results from a position $z$ vs. losses tradeoff. ${ }^{20}$ We note here, that $10 \mathrm{~nm}$ of copper and one groove are enough to increase transmission to the level of a much larger aperture. Naturally, a thicker layer pushes the enhancement to even higher values, although at a cost of decreasing resolution. For $10 \mathrm{~nm}$ of $\mathrm{Cu}$ resolution is approximately the same as of a pure $\mathrm{Al} r=15 \mathrm{~nm}$ probe (better than $50 \mathrm{~nm}$ ) with the exception
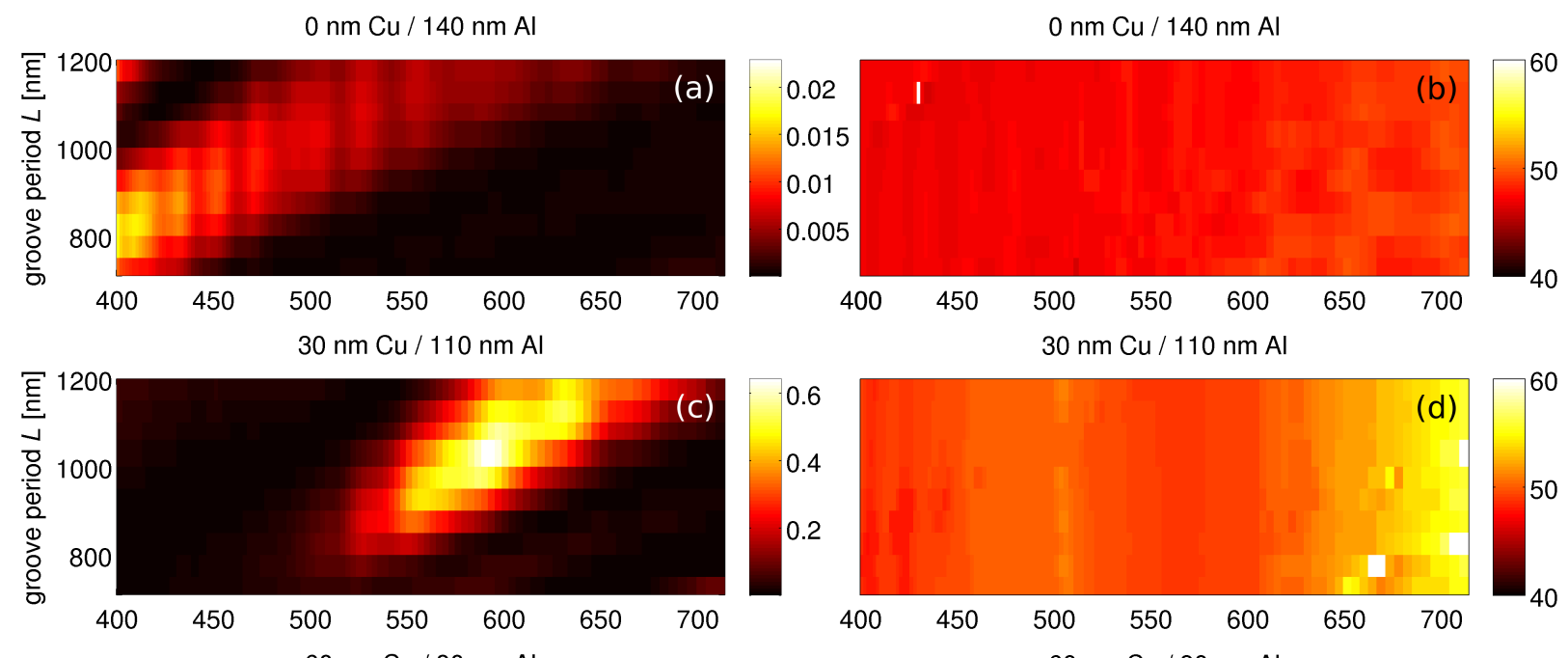

$60 \mathrm{~nm} \mathrm{Cu} / 80 \mathrm{~nm} \mathrm{Al}$
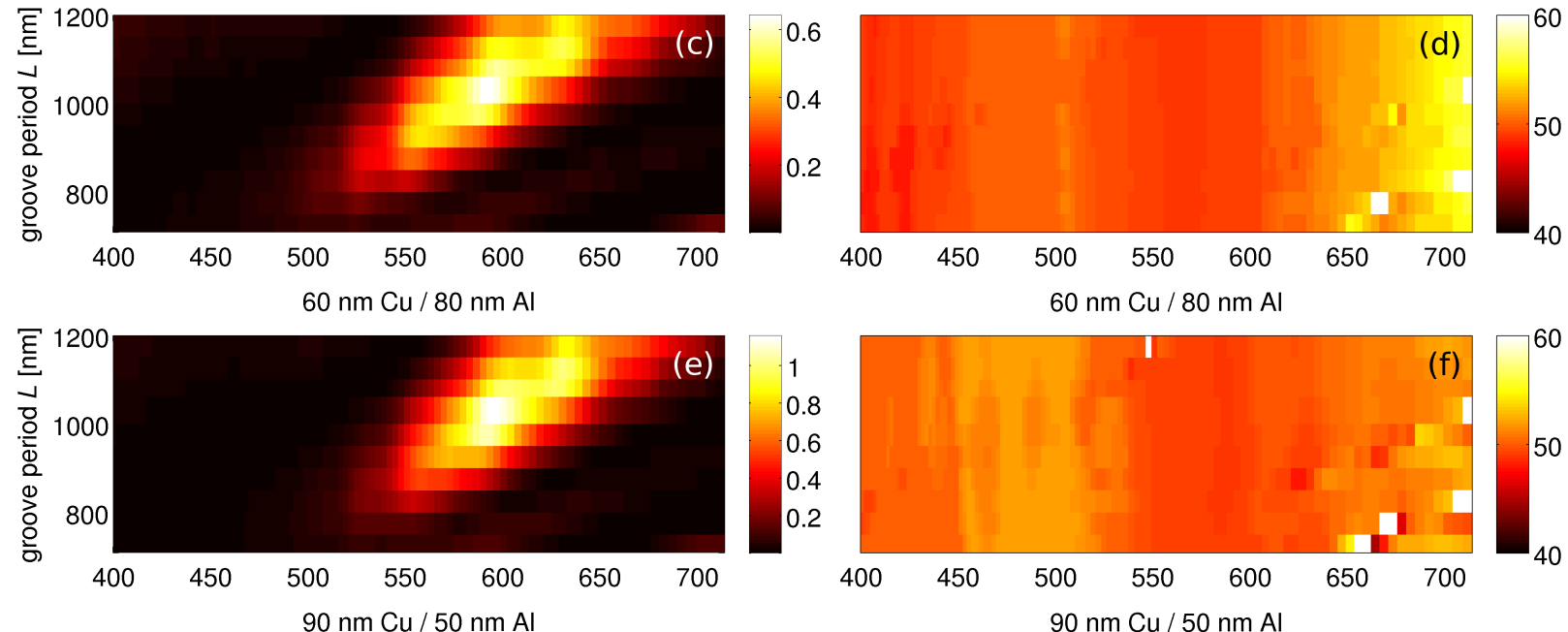

$60 \mathrm{~nm} \mathrm{Cu} / 80 \mathrm{~nm} \mathrm{Al}$
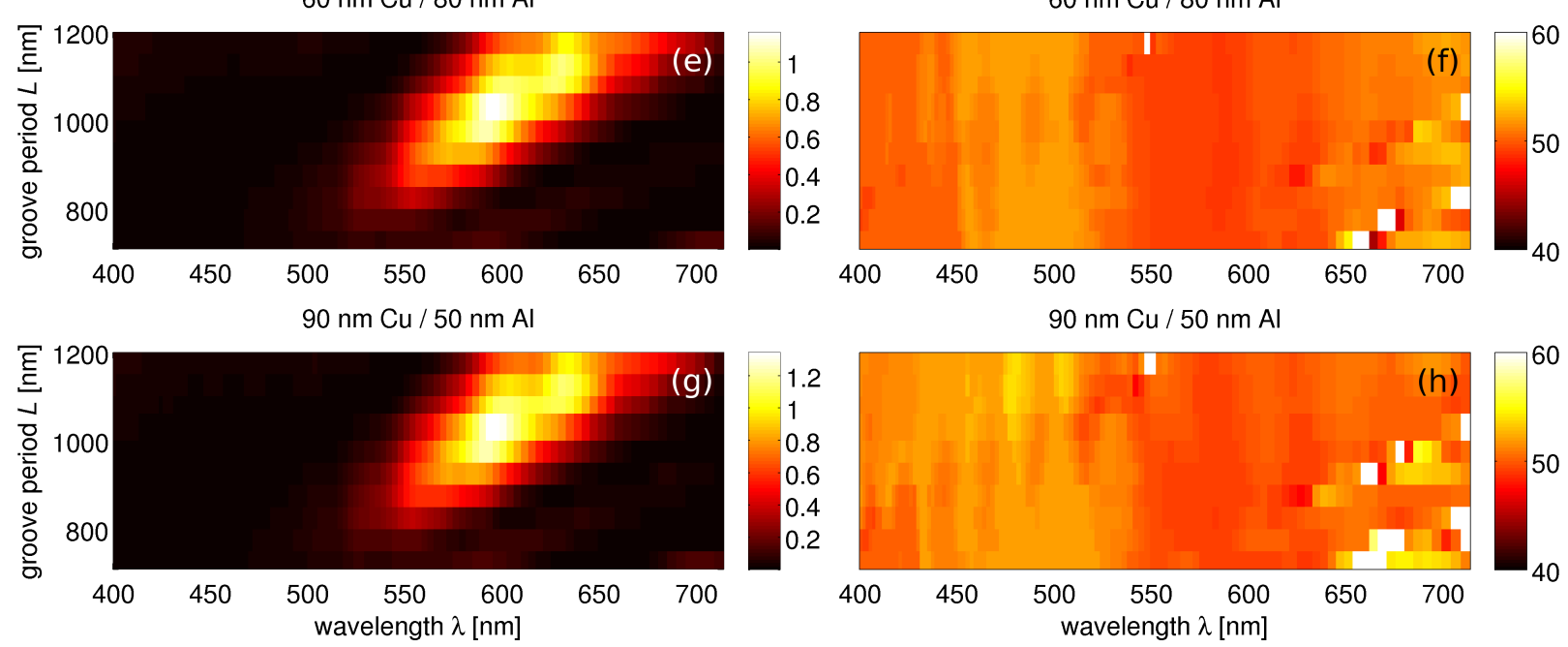

Figure 6. Transmission (a), (c), (e), (g) and resolution (b), (d), (f), (h) of Cu/Al coated probes $(r=15 \mathrm{~nm})$ with a sinusoidal groove lattice as function of groove periodicity $L$ for wavelengths $\lambda \in[400 ; 700] \mathrm{nm}$. Copper thickness $h_{i}$ is $0 \mathrm{~nm}$ in (a), (b), $30 \mathrm{~nm}$ in (c), (d), $60 \mathrm{~nm}$ in (e), (f), and $90 \mathrm{~nm}$ in (g), (h). Transmission is normalized to that of a pure $\mathrm{Al}$ coated unstructured $r=25 \mathrm{~nm}$ probe. 
of wavelengths larger than $630 \mathrm{~nm}$. For greater thicknesses resolution is decreased by approximately $5 \mathrm{~nm}$ (up to about $55 \mathrm{~nm}$ ), what is still better than for the larger probe with offers $70 \mathrm{~nm}$ resolution.

\subsection{Probes with a sinusoidal grating}

The second type of structure at the core-metal interface is a sinusoidal grating which can be fabricated by etching optical fibers with a distributed Bragg reflector (DBR) written in the core. We have not yet carried out a full numerical investigation of such gratings, so the results presented here are not optimised and serve only to indicate possibilities offered by combining a bi-metal cladding with a DBR probe. However, we have analysed previously other types of gratings ${ }^{17,18}$ and those general results are valid for these modified gratings, though not exactly. Here, in addition to the inner metal thickness $h_{i}$ we vary the groove periodicity $L$.

Figure 6 presents transmission enhancement and resolution of $\mathrm{Cu} / \mathrm{Al}$ coated probes with a sinusoidal grating of the core-coating interface. As with single-groove probes, these results are normalized to those of a pure Al probe with an aperture radius of $r=25 \mathrm{~nm}$. Pure Al coated probes (Fig. 6a,b) have poor energy efficiency, as expected, but offer resolution on the order of $48 \mathrm{~nm}$. When the copper layer thickness increases transmission increases and the maximum redshifts due to dispersion (see the imaginary part of permittivity in Fig. 3), but is accompanied by a decrease of resolution by $3 \mathrm{~nm}$. However, in the spectral range where transmission is large, the spot size is about the same as for a pure Al probe. To match the efficiency of larger probes we need to have at least $50 \mathrm{~nm}$ copper. This change increases transmission - the increase is the larger the thicker is the copper layer, but does not worsen resolution within the area of large transmission while $h_{i}<100 \mathrm{~nm}$.

\subsection{Impact of an inner metal layer}

An addition of a noble metal layer between the core and the outer aluminum cladding allows for and increase of transmission. This increase translates into a shrinckage of the aperture diameter and resolution enhancement. Use of two layers (with the outer being aluminum) has an advantage over pure noble metal cladding that it limits tunneling of light through the coating. It keeps resolution from decreasing due to a strong signal on the outside metal - surrounding medium interface, what can be observed for pure low-loss metal coatings. While the increase due to a bi-layer and corrugations was shown here only for copper, similar results are observed for gold and silver, though in the latter case the working wavelength range is somewhat limited.

\section{CONCLUSIONS}

In this report we analysed the impact of a bilayer consisting of an inner low dissipation metal $(\mathrm{Ag}, \mathrm{Au}, \mathrm{Cu})$ layer and an outer aluminum layer on the transmission efficiency and resolution of aperture SNOM probes. The analysis was carried out using BOR-FDTD simulations in which we analyzed transmission spectra of various kinds of probes without and with corrugations of the core-coating interface. Results show that corrugations together with an inner copper layer allow for a considerable resolution increase while keeping the energy throughput at a comparable level to that of a probe with a larger spot size.

\section{ACKNOWLEDGMENTS}

This work was supported by the Polish Ministry of Science and Higher Education under the project Iuventus Plus 0480/H03/2010/70 and the National Centre for R\&D under the project N R15 0018 06. The authors are partners in COST Actions MP 0702 and MP 0803. Simulations were performed at the Interdisciplinary Centre for Mathematical and Computational Modelling at the University of Warsaw under grant \#G33-7.

\section{REFERENCES}

[1] Pohl, D., Denk, W., and Lanz, M., "Optical stethoscopy: Image recording with resolution $\lambda / 20$," Appl. Phys. Lett. 44, 651-653 (1984).

[2] Burresi, M., van Oosten, D., Kampfrath, T., Schoenmaker, H., Heideman, R., Leinse, A., and Kuipers, L., "Probing the magnetic field of light at optical frequencies," Science 326, 550-553 (2009).

[3] Antosiewicz, T. J., Wróbel, P., and Szoplik, T., "Magnetic field concentrator for probing optical magnetic metamaterials," Opt. Express 18, 25906-25911 (2010). 
[4] Burresi, M., Kampfrath, T., van Oosten, D., Prangsma, J., Song, B., Noda, S., and Kuipers, L., "Magnetic light-matter intersactions in a photonic crystal nanocavity," Phys. Rev. Lett. 105, 123901 (2010).

[5] Vignolini, S., Intonti, F., Riboli, F., Balet, L., Li, L., Francardi, M., Gerardino, A., Fiore, A., Wiersma, D., and Gurioli, M., "Magnetic imaging in photonic crystal microcavities," Phys. Rev. Lett. 105, 123902 (2010).

[6] Whitesides, G., "The 'right' size in nanobiotechnology," Nature Biotechnol. 21, 1161-1165 (2003).

[7] Klar, T., Perner, M., Grosse, S., von Plessen, G., Spirkl, W., and Feldmann, J., "Surface-plasmon resonances in single metallic nanoparticles," Phys. Rev. Lett. 80, 4249-4252 (1998).

[8] Stöckle, R., Suh, Y., Deckert, V., and Zenobi, R., "Nanoscale chemical analysis by tip-enchanced Raman spectroscopy," Chem. Phys. Lett. 318, 131-136 (2000).

[9] Ashkin, A., "Optical trap and manupulation of neutral particles using lasers," PNAS 94, 4853-4860 (1997).

[10] Klimov, V., Sekatskii, S., and Dietler, G., "Laser nanotraps and nanotweezers for cold atoms: 3D gradient dipole force trap in the vicinity of scanning near-field optical microscope tip," Opt. Commun. 259, 883887 (2006).

[11] Yang, A., Moore, S., Schmidt, B., Klug, M., Lipson, M., and Erickson, D., "Optical manupulation of nanoparticles and biomolecules in sub-wavelength slot waveguides," Nature 457, 71-75 (2009).

[12] Wang, Z., Cai, X., Chen, Q., and Li, L., "Optical properties of metal-dielectric multilayers in the near UV region," Vacuum 80, 438-443 (2006).

[13] Imura, K., Ueno, K., Misawa, H., and Okamoto, H., "Anomalous light transmission from plasmoniccapped nanosapertures," Nano Lett. 11, 960-965 (2011).

[14] Yatsui, T., Kourogi, M., and Ohtsu, M., "Increasing throughput of a near-field optical fiber probe over 1000 times by the use of a triple-tapered structure," Appl. Phys. Lett. 73, 2090-2092 (1998).

[15] Murphy-DuBay, N., Wang, L., and Xu, X., "Nanolithography using high transmission nanoscale ridge aperture probe," Appl. Phys. A 93, 881-884 (2008).

[16] Tanaka, K., Tanaka, M., and Sugiyama, T., "Creation of strongly localized and strongly enhanced optical near-field on metallic probe-tip with surface plasmon polaritons," Opt. Express 14, 832-846 (2006).

[17] Antosiewicz, T. and Szoplik, T., "Corrugated metal-coated tapered tip for scanning near-field optical microscope," Opt. Express 15, 10920-10928 (2007).

[18] Antosiewicz, T. and Szoplik, T., "Corrugated SNOM probe with enhanced energy throughput," OptoElectron. Rev. 16, 451-457 (2008).

[19] Wang, Y., Srituravanich, W., Sun, C., and Zhang, X., "Plasmonic nearfield scanning probe with high transmission," Nano Lett. 8, 3041-3045 (2008).

[20] Antosiewicz, T., Wróbel, P., and Szoplik, T., "Performance of scanning near-field optical microscope probes with single groove and various metal coatings," Plasmonics 6, 11-18 (2011).

[21] Taflove, A. and Hagness, S., [Computational electrodynamics], Artech House, Norwood, MA, third ed. (2005).

[22] Antosiewicz, T., Wróbel, P., and Szoplik, T., "Nanofocusing of radially polarized light with dielectricmetal-dielectric probe," Opt. Express 17, 9191-9196 (2009).

[23] Johnson, P. and Christy, R., "Optical constants of the noble metals," Phys. Rev. B 6, 4370-4379 (1972).

[24] Ordal, M., Long, L., Bell, R., Bell, R., R.W. Alexander, J., and Ward, C., "Optical properties of the metals Al, $\mathrm{Co}, \mathrm{Cu}, \mathrm{Fe}, \mathrm{Pb}, \mathrm{Ni}, \mathrm{Pd}, \mathrm{Pt}, \mathrm{Ag}$, Ti, and $\mathrm{W}$ in the infrared and far infrared," Appl. Opt. 22, 1099-1119 (1983). 